\title{
Regret and the negative evaluation of decision outcomes in major depression
}

\author{
Henry W. Chase \\ University of Cambridge, Cambridge, England \\ Nathalie Camille \\ University of Cambridge, Cambridge, England \\ and Mc Gill University, Montreal, Quebec, Canada \\ Albert Michael \\ West Suffolk Hospital, Bury St Edmunds, England \\ EdWARd T. BULLMORE AND TREVOR W. RobBins \\ University of Cambridge, Cambridge, England \\ AND \\ Barbara J. SAHAKiaN \\ University of Cambridge School of Clinical Medicine, Cambridge, England
}

\begin{abstract}
Disruption of normal emotional experience is central to the phenomenology of depression. Twenty-three depressed outpatients and 23 control subjects performed a computerized decision-making task, during which affective ratings were assessed online to identify various dimensions of emotional experience. We sought to contrast regret (the comparison of the outcomes of selected and nonselected options) with the general negative appraisal of task events. The experience of regret was reduced in depressed patients, an effect that was particularly related to self-reported apathy scores. In an exploratory analysis, we observed that females had a general downward shift in their ratings, as compared with males, but disappointment and regret effects were of similar magnitude. The possible contribution of the orbitofrontal cortex to the phenomenology of regret is discussed. Supplemental materials for this article may be downloaded from http://cabn.psychonomic-journals.org/content/ supplemental.
\end{abstract}

Depression is associated with a disruption of normal emotional experience, the phenomenology of which can be divided into two broad categories: a negative bias - the tendency to appraise events, both forthcoming and past, negatively - and anhedonia, which includes a "blunting" of the normal experience of reward (Chase et al., 2009; Pizzagalli, Jahn, \& O'Shea, 2005; Steele, Kumar, \& Ebmeier, 2007). What is less clear is the manner in which these two symptoms might interact to influence the emotional appraisal of salient events, including disappointment and regret, since our understanding of the dynamics of these emotions (e.g., Larsen, McGraw, Mellers, \& Cacioppo, 2004) has advanced beyond our theoretical understanding of emotional changes in depression.

The utility of an outcome, which has a strong influence on the emotional response engendered by it (Mellers, 2000), has been argued to depend on reference points (Kahneman \& Tversky, 1979), such that a positive movement compared with a low reference point might be evaluated positively, even if its absolute value is negative. Hence, if one assumes that the reference point is modified by depression, one might come to very different conclusions about the consequential emotional response to an outcome than if one assumes that the reference point is similar but the response to the outcome itself is modified. Notably, in order to demonstrate greater disappointment in depression, one must assume that the reference point is actually higher in depressed patients; that is, states of the world that they would consider possible during outcome evaluation are more rewarding than those considered by control participants. This seems to be a counterintuitive prediction, since generally, depressed patients are thought to have a negative evaluation of the world (Beck, 1967). However, Knutson, Bhanji, Cooney, Atlas, and Gotlib (2008) observed enhanced anticipation-related neural activation prior to rewarding events in a group of depressed subjects, as compared with controls.

Another view is that feedback might be less emotive in depressed patients, causing a "blunting" effect, in which

H.W. Chase, henry.chase@nottingham.ac.uk 
patients show a particular insensitivity to positive feedback (Pizzagalli et al., 2005) or a generalized reduction in sensitivity to feedback regardless of valence (e.g., Chase et al., 2009; Steele et al., 2007). A number of neuroimaging experiments have been designed to evaluate the fundamental aspects of reward and punishment processing. Reduced neural activation elicited by rewards in regions of the medial frontal cortex and striatum has been observed (Knutson et al., 2008; Kumar et al., 2008; Pizzagalli et al., 2009). Consistent with a negative bias, enhanced punishment or loss-related activity has also often been observed, particularly in studies employing event-related potentials (e.g., Chiu \& Deldin, 2007; Holmes \& Pizzagalli, 2008), but equally so has been attenuated loss-related activity (Steele et al., 2007).

We also considered the possibility that the emotion of regret might be selectively modulated by depression, as has been suggested by Coricelli, Dolan, and Sirigu (2007; see also Monroe, Skowronski, MacDonald, \& Wood, 2005), who suggested, given the propensity of those with depression to experience negative emotions, that the experience of regret should be greater in these individuals. However, a different perspective might also be entertained. Zeelenberg, van Dijk, Manstead, and van der Pligt (2000) emphasized the dimension of instrumentality as a means of distinguishing them. Specifically, regret is dependent on the notion that an alternative situation might have occurred had the individual acted differently. By contrast, disappointment might be said to arise rather directly from life events, over which occurrence one has little control. The experience of regret is considered to have an adaptive role, informing future behavior, whereas the anticipation of regret may also have an important role in decision making (Roese, 1997). Representation of the contribution of action to decision making might be rendered somehow ineffective or irrelevant by apathy, which perhaps results in symptoms of hopelessness (Abramson, Metalsky, \& Alloy, 1989) and/or apathy observed in depression. We therefore predicted that regret would be reduced in depression, in a manner that would depend on self-reported apathy scores, measured using the Apathy Evaluation Scale-Self (AES-S; Marin, Biedrzycki, \& Firinciogullari, 1991).

We adapted existing behavioral paradigms to investigate these effects (Camille et al., 2004; Rogers et al., 1999). Briefly, the subjects were presented with two options, the relative risk and the expected value (EV) of which were manipulated. During each trial, affective ratings were assessed on three occasions: First, the subjects were asked to rate the quality of their decision just after making a decision but before receiving feedback; second, the subjects rated their affective response to discovering the outcome of their decision; third, the subjects rated their affective response to discovering the outcome of the nonselected option. The same statements ( pleased/disappointed) were used to define the ratings scale at the second and third rating steps. Hence, the focus of the analysis and the consequent inference was on the conditions (i.e., the relative value of the nonobtained and obtained outcomes) required to elicit a given movement of affective rating, rather than being dependent on the nature of the rating scale statements themselves.

We were then in a position to test several hypotheses: that depressed patients would show a generalized attenuated effect to reinforcement, which would be evident if the degree of modulation of affective responses by wins or losses was reduced in the patient group, as compared with controls; that depressed patients would show a negative bias, rating the outcomes of a decision more negatively than would controls; and finally, that these effects might be differentially reflected in the emotional responses to the outcome of the selected option and the outcome of the nonselected option (i.e., a specific modification of regret).

In order to examine the affective response to the obtained outcome, we collapsed trials on which the outcome was as good as or better than the EV of the option and compared it with trials on which the outcome was worse than the EV of the option. In order to examine regret, we focused on the final (third) rating, collapsing trials on which the nonobtained outcome was greater than the obtained outcome, and compared them with trials on which the obtained outcome was greater than or equal to the nonobtained outcome. The latter measure represents a useful metric of regret: Although it is perhaps less direct than asking the subjects directly to assess their experience of regret, it may also be consequently less contaminated by the negative connotations the word possesses, which might precipitate uncontrolled group differences. We also analyzed different parameters of decision-making performance to identify whether there were systematic differences in the quality or riskiness of the choices of patients and controls.

\section{METHOD}

\section{Subjects}

Twenty-three outpatients (13 males) who met the DSM-IV (American Psychiatric Association, 1994) criteria for major depressive disorder participated in this study. Patients with other DSM-IV Axis 1 disorders and with neurological or general medical disorders likely to affect cognition were excluded. In addition, patients treated with ECT in the previous year and those prescribed benzodiazepines or antipsychotic drugs were also excluded. Twenty-three healthy matched controls (12 males) were recruited from the local community. The controls had no history of psychiatric or neurological disease and were group-matched to the patient group for age and gender. The National Adult Reading Test (Nelson \& Willison, 1991), a measure of (premorbid) intellectual function, was administered to all the subjects. All of the subjects provided informed consent and were paid for participation. This study was approved by the Charing Cross/Cambridgeshire 2 and Suffolk Local Research Ethics Committees.

Of the 23 patients, 3 were not on antidepressants ( 2 were not medicated at all), 14 patients received a single antidepressant, and 6 took two or more in combination. Twelve took selective serotonin reuptake inhibitors (SSRIs), 3 took selective serotonin and noradrenaline reuptake inhibitors (SNRIs), 5 took tricyclic antidepressants, 5 took trazodone, and 1 patient took bupropion. Six patients were also on mood stabilizers, 2 were on propranolol, and 4 were taking opiate analgesics. The medications were continued unchanged during the study. 


\section{Questionnaire Measures}

Severity of depression was assessed in both groups using the Beck Depression Inventory (BDI; Beck, Ward, \& Mendelson, 1961), and controls with a BDI score of 9 or over were excluded. Depressed patients were also administered the Montgomery-Asberg Depression Rating Scale (MADRS; Montgomery \& Asberg, 1979). The Beck Anxiety Inventory (BAI; Beck, Epstein, Brown, \& Steer, 1988) was administered in order to assess the level of anxiety symptoms. In line with predictions, data for each subject on the AES-S (Marin et al., 1991) were also obtained. For the latter questionnaire, subfactor scores, reflecting behavioral, emotional, and cognitive aspects of apathy, were also calculated (Marin, 1996). There were some missing questionnaire data for the AES-S: Five subjects had one missing data point, 1 subject had two missing data points, and 2 patients were missing the whole questionnaire. If data points were missing, the average score per question for the questions that the subject completed within a subfactor was calculated and added to the subject's score for each missing question.

\section{Regret Task Procedure}

The task followed the presentational structure employed by Rogers et al. (1999) but included measurement of affective responses. The task was administered on a portable PC with a touchscreen. The subjects were presented with a screen that displayed two options on either side of the screen. The possible outcomes, and their associated probabilities, of selecting each option also were displayed (a screen shot of the task is presented in the supplementary materials). Each choice had two possible outcomes, displayed above and below a rectangular bar. The outcome below the bar was always zero; the outcome above the bar was either a reward $(10,20,40$, or 60 points) or a punishment $(-60,-40,-20$, or -10 points; Table 1 contains further details about the design matrix). On a given trial, both outcomes above the bar were either rewards (18 trials) or punishments (10 trials), but never one of each. The colors of the bar represented the relative probability of the outcome above or below the bar: The proportion of green on the bar represented the likelihood of the option above the bar, and the proportion of blue represented the likelihood of the option below the bar. The proportion of green could be $100 \%, 75 \%, 50 \%$, or $25 \%$. Task order was fully randomized, and a random number generator was also used to determine the outcomes, given the stated probabilities presented in the task. The subjects selected a given option by pressing a button below the option. They made ratings about their decision making on a visual analogue scale (VAS) presented on the bottom half of the screen. When making a selection, the subjects would touch the screen around the VAS at a point that corresponded with their rating, leaving a marker on the screen. If they were unhappy with the position of their rating, they could change its position as many times as they liked, until they pressed a central button to continue with the task.

Each trial followed the same pattern.

1. The subject selected one of the two options.

2. The subject rated the quality of his or her decision (quality rating). The rating to be made was "Rate your confidence that you made the right decision." On the far left-hand side of the rating scale, the marker was labeled "Bad Choice"; on the far right-hand side, the marker was labeled "Good Choice"; in the center, the marker was labeled "Completely Uncertain." The subject pressed the button in the center of the screen to continue when happy with the rating.

3. The subject saw the outcome of his or her choice and made another rating: "Rate feelings about the consequences of your choice." On the far left-hand side of the rating scale, the marker was labeled "Extremely Disappointed"; on the far right-hand side, the marker was labeled "Extremely Pleased"; in the center, the marker was labeled "Neither Pleased nor Disappointed." The outcome was displayed on the screen by a marker appearing next to the probability bar denoting which of the two outcomes was obtained. A green box surrounded the obtained outcome. The subject pressed the continue button.

4. The subject saw the outcome of the option that he or she had not selected and then made the same rating as that in the previous round in the light of this new information. A marker appeared next
Table 1

Task Design Matrix

\begin{tabular}{|c|c|c|c|}
\hline LHS Value & $\begin{array}{c}\text { LHS } \\
\text { Probability }\end{array}$ & RHS Value & $\begin{array}{c}\text { RHS } \\
\text { Probability }\end{array}$ \\
\hline
\end{tabular}

\begin{tabular}{|c|c|c|c|}
\hline \multicolumn{4}{|c|}{ Win Trials } \\
\hline 10 & 1.00 & 40 & .25 \\
\hline 10 & 1.00 & 20 & .50 \\
\hline 10 & 1.00 & 40 & .50 \\
\hline 20 & .75 & 60 & .25 \\
\hline 20 & .50 & 40 & .25 \\
\hline 20 & .75 & 40 & .50 \\
\hline 20 & 1.00 & 40 & .50 \\
\hline 20 & .50 & 10 & 1.00 \\
\hline 40 & .50 & 60 & .25 \\
\hline 40 & .25 & 20 & .50 \\
\hline 40 & 1.00 & 60 & .50 \\
\hline 40 & .50 & 20 & .75 \\
\hline 40 & .25 & 10 & 1.00 \\
\hline 40 & .50 & 10 & 1.00 \\
\hline 40 & .50 & 20 & 1.00 \\
\hline 60 & .25 & 40 & .50 \\
\hline 60 & .25 & 20 & .75 \\
\hline 60 & .50 & 40 & 1.00 \\
\hline \multicolumn{4}{|c|}{ Loss Trials } \\
\hline-60 & .50 & -40 & 1.00 \\
\hline-40 & 1.00 & -60 & .50 \\
\hline-40 & .50 & -10 & 1.00 \\
\hline-40 & .25 & -10 & 1.00 \\
\hline-40 & .50 & -20 & 1.00 \\
\hline-20 & 1.00 & -40 & .50 \\
\hline-20 & .50 & -10 & 1.00 \\
\hline-10 & 1.00 & -40 & .25 \\
\hline-10 & 1.00 & -20 & .50 \\
\hline-10 & 1.00 & -40 & .50 \\
\hline
\end{tabular}

Note-Each row represents the components of an individual trial. Loss and win trials were intermingled, and the order of all trials was randomized between subjects. "Value" and "Probability" columns reflect the value of the winning outcome and its probability on a given side. LHS, left-hand side; RHS, right-hand side.

to the probability bar in the same way as with the obtained outcome, and a red box surrounded the nonobtained outcome. The subject pressed the continue button and the next trial began. At four points during the task, the subject was given a running total of his or her score at that stage.

Two practice trials at the start of the procedure were included, but data from these trials were not analyzed. During the practice session, the subjects were instructed that the purpose of the task was to earn points and avoid losing points. The nature of the probabilities and reward values, the way in which feedback about the task would be presented, and the manner in which the VAS should be used, particularly that a rating could be made at any point on the line, were explained to the subject.

\section{Data Analysis}

Ratings were derived by rescaling the subjects' touch position along the VAS between 1 and -1 . Ratings that were slightly outside the scale, due to an inaccurate response, were bounded at 1 or -1 , depending on their side. The scope for this possibility was limited, and unbounding the ratings had virtually no effect on the results. Due to computer errors, 4 subjects were missing a single affective data point each (third rating).

To investigate emotional responses to the obtained outcome, we collapsed the ratings for the obtained outcome of trials on which the outcome of the selected option was equal to or higher than the EV of the option (value of the maximum available amount multiplied by its probability). We also extracted ratings for trials on which the outcome of the selected option was less than the EV. These were in- 
Table 2

Demographic Variables for Patients and Controls

\begin{tabular}{lcccccc}
\hline & \multicolumn{2}{c}{$\begin{array}{c}\text { Patients } \\
(n=23)\end{array}$} & & \multicolumn{2}{c}{$\begin{array}{c}\text { Controls } \\
(n=23)\end{array}$} & \\
\cline { 2 - 3 } \cline { 5 - 6 } Variable & $M$ & $S E$ & & $M$ & $S E$ & $t$ Statistic \\
\hline Age & 46.22 & 2.25 & & 47.74 & 2.14 & $t(44)<1$ \\
NART errors & 18.43 & 2.19 & & 16.52 & 1.74 & $t(44)<1$ \\
BDI & 26.78 & 1.79 & & 3.17 & 0.49 & $t(25.3)=12.690, p<.001$ \\
MADRS & 26.17 & 1.50 & & N/A & - & \\
BAI & 32.39 & 2.35 & & 5.61 & 0.88 & $t(28.1)=10.665, p<.001$ \\
AES-S & 46.82 & 1.56 & & 62.96 & 0.91 & $t(42)=9.086, p<.001$ \\
\hline
\end{tabular}

Note-Differences between Beck Depression Inventory (BDI), Beck Anxiety Inventory (BAI), and Apathy Evaluation (AES) scores for the two groups were highly significant, but not those for age or the National Adult Reading Test (NART). Montgomery-Asberg Depression Rating Scale (MADRS) data were not obtained for control subjects.

serted into a repeated measures ANOVA, where group (patients vs. controls) was included as a between-subjects factor. Quality ratings were analyzed using a one-way ANOVA, with group as a betweensubjects factor.

Likewise, to investigate regret, we collapsed ratings for the nonobtained outcome on trials on which the nonobtained outcome was greater than the obtained outcome with those for trials on which it was less than or equal to the obtained outcome. These were similarly analyzed using a repeated measures ANOVA with group as a between-subjects factor. Post hoc $t$ tests were performed to investigate the magnitude of the regret effect in each group.

Decision-making performance was investigated using two repeated measures ANOVAs. In the first model, the distribution of subjects' choices on trials on which the two options were matched for EV but had different amounts of risk (one option led to a certain outcome, the other led to an uncertain outcome) were extracted separately for decisions on which the EVs of the options were positive and negative. In the second, choices on trials in which the EVs of the outcomes were not matched were extracted: The proportions of choices where the EVs were 5 points higher and 10 points higher were extracted separately. The latter data were also analyzed with a one-sample $t$ test, to check whether the subjects were likely to select options with a higher EV or whether they were responding randomly.

Demographic characteristics and questionnaire data were analyzed using a two-sample $t$ test, examining the effect of group (patients vs. controls). The data are presented in Table 2. An alpha level of .05 was used in all planned statistical comparisons. The GreenhouseGeisser correction was applied when the homogeneity of variances was violated.

\section{RESULTS}

\section{Quality Ratings and Emotional Responses to the Outcome}

A univariate ANOVA was performed on the quality ratings, with the contrast between patients and controls as a between-subjects factor. No significant main effect of group was observed $\left[F(1,44)=2.738, p=.105, \eta_{\mathrm{p}}^{2}=\right.$ .059 ; see Figure 1]. Affective ratings for the outcome were split into ratings made when the obtained outcome was as good as or better than the EV (wins) versus when the obtained outcome was worse than the EV (losses) and were inserted into a repeated measures ANOVA. Ratings for wins were greater than ratings for losses $[F(1,44)=94.639$, $\left.p<.001, \eta_{\mathrm{p}}^{2}=.683\right]$. The main effect of group was not significant $\left[F(1,42)=2.053, p=.159, \eta_{\mathrm{p}}^{2}=.045\right]$, nor was the interaction term significant $\left[F(1,44)<1, \eta_{\mathrm{p}}^{2}=\right.$ $.003]$. In summary, responses to the value and expectancy of the obtained outcome were similar in both groups (see Figure 1).

\section{Regret}

Regret was evaluated by comparing trials on which the nonobtained outcome was greater than expected versus those on which it was less than expected. We observed a main effect of regret versus relief $[F(1,44)=15.690$, $\left.p<.001, \eta_{\mathrm{p}}^{2}=.263\right]$ and a group $\times$ regret interaction $\left[F(1,44)=4.884, p=.032, \eta_{\mathrm{p}}^{2}=.100\right]$ but no main effect of group $\left[F(1,44)<1, \eta_{\mathrm{p}}^{2}=.014\right.$; see Figure 2]. Post hoc $t$ tests were used to evaluate this effect further. The overall magnitude of the regret effect (final ratings when the nonobtained outcome is less than or equal to the obtained outcome minus final ratings when the obtained outcome is less than the nonobtained outcome) was greater for controls than for patients $[t(44)=2.210, p=.032]$. The two subcomponents of this effect were examined. For controls, ratings where the obtained outcome was less than the nonobtained outcome were significantly less than zero

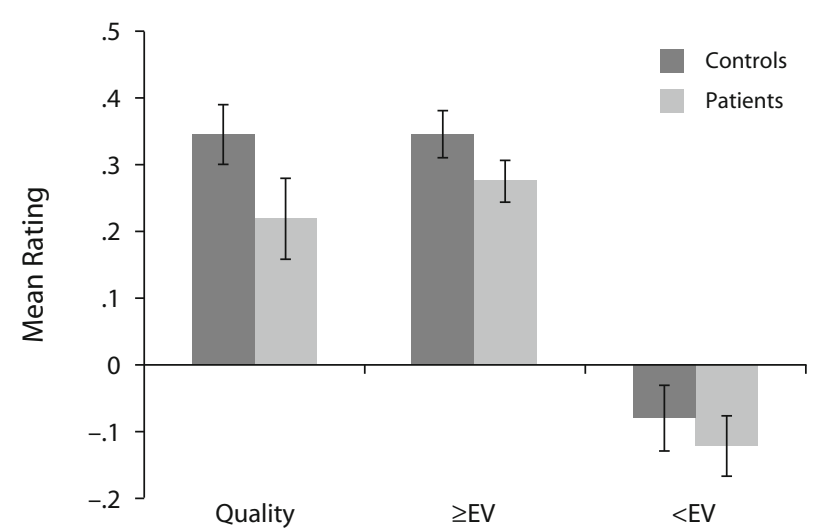

Rating Type

Figure 1. Quality ratings and selected outcome ratings (split by whether the outcome was greater than or equal to the expected value [EV] vs. less than the EV) for controls and patients. Error bars represent standard errors of the means. 


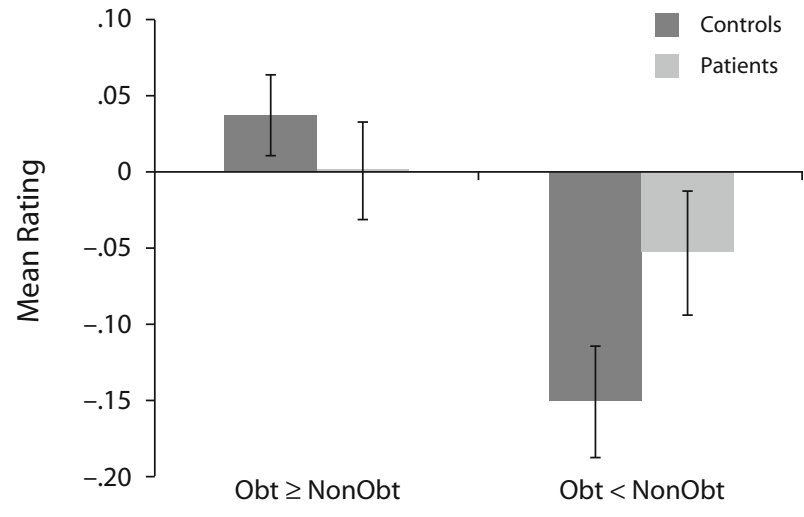

Rating Type

Figure 2. Ratings for the value of the nonselected outcome, split by whether the obtained outcome was greater than or equal to the nonobtained outcome or whether it was less than the nonobtained outcome, for controls and patients. Error bars represent standard errors of the means.

$[t(22)=-4.140, p<.001]$, and where the obtained outcome was greater than the nonobtained outcome, ratings were positive, although not significantly greater than zero $[t(22)=1.420, p=.170]$. For patients, neither of the two contrasts was significantly different from zero [nonobtained $>$ obtained, $t(22)=-1.319, p=.201$; obtained $>$ nonobtained, $t(22)<1]$.

\section{Effect of Apathy on Regret}

Self-reported ratings of apathy correlated with the difference between the nonobtained outcome and the obtained outcome $(r=-.411, n=44, p=.006)$ : Subjects who showed higher apathy scores showed a reduced regret effect. We also investigated the effect of different dimensions of apathy: Regret was best associated with self-reported "emotional" apathy $(r=-.474, n=44, p=$ $.001)$, and less so with "cognitive" $(r=-.320, n=44$, $p=.034)$ and "behavioral" $(r=-.357, n=44, p=.017)$ apathy. Neither total apathy score nor any of the subscales were significantly correlated with affective responses to win versus loss events ( $p>.123$ in all cases).

\section{Decision Making}

Percentages of risky choices for trials that were controlled for EV ( 10 choices between wins; 6 choices between losses) were inserted into a repeated measures ANOVA (see Figure 1). There was no main effect of frame: Risk taking was no more likely between losses than between wins $\left[F(1,44)=1.872, p=.178, \eta_{\mathrm{p}}^{2}=.041\right]$. The interactions between framing and group and the main effect of group were not significant $\left[F(1,44)<1, \eta_{\mathrm{p}}^{2}<.016\right.$, in both cases]. No significant bias was observed toward or away from the riskier option in either group, in either frame.

Percentages of choices of options with higher EV than the alternative were split where the difference in EV was 5 or 10 points and were inserted into a repeated measures ANOVA. There were no main effects of EV difference or group and no interaction between the two $[F(1,44)<1$ in all cases]. Selection of the higher EV option was significantly greater than chance for both groups in both EV conditions $[t(22)>2.732, p<.012$, in all cases].

Partly as a consequence of the matching of decisionmaking performance between the two groups, the distribution of outcome values was also well matched. The difference between the mean values on win trials and loss trials was highly significant $[F(1,44)=648.029, p<.001]$, but the main effect of group and the interaction between win/ loss and group were not significant $[F(1,44)<1$ in both cases]. Likewise, the value of the nonselected outcome was greater on trials on which it was greater than the obtained outcome than on trials on which it was less than the obtained outcome [both corrected for the value of the obtained outcome: $F(1,44)=4,723.632, p<.001]$, but the interaction of greater/less than the obtained outcome and group and the main effect of group were both not significant $[F(1,44)<1$, in both cases]. Similar null effects were observed for the effect of gender on the outcome parameters.

\section{The Effect of Gender}

As an exploratory analysis, we also investigated the effect of gender (male vs. female). Quality ratings were more negative for females than for males $[F(1,45)=$ $\left.8.264, p=.006, \eta_{\mathrm{p}}^{2}=.158\right]$. There was also a main effect of gender on emotional responses to the outcome $\left[F(1,44)=5.551, p=.023, \eta_{\mathrm{p}}^{2}=.112\right]$ but no gender $\times$ win/loss interaction $\left[F(1,44)<1, \eta_{\mathrm{p}}^{2}=.003\right]$, again with females showing more negative ratings than did males. There was also a main effect of gender on ratings following the nonselected outcome, once corrected for the value of the obtained outcome $\left[F(1,44)=8.400, p=.006, \eta_{\mathrm{p}}^{2}=\right.$ $.160]$, but no effect on the magnitude of the regret/relief effect $\left[F(1,44)<1, \eta_{\mathrm{p}}^{2}=.006\right]$. However, this was in the opposite direction (males $<$ females) and was a result of the correction procedure (see the Method section). These results represent a distinct pattern, as compared with the effects of depression: The magnitude of emotional responses to obtained outcomes and regret was similar, but there was a negative shift in quality and obtained outcome ratings in females, as compared with males.

\section{Summary}

Control subjects showed a modulation of affective responses on trials on which the outcome of the nonselected option was greater than the outcome of the selected option, as compared with trials on which the outcome of the selected option was greater than or equal to the nonselected option. This effect was significantly smaller in depressed patients and was associated with individual differences in apathy. Depression did not have a clear effect on the ability of rewards to modulate emotion or on parameters of decision making. The pattern of data suggests that depressed patients show a reduction in the experience of regret.

\section{DISCUSSION}

In this study, the pattern of decision making and affective responses during decision making was assessed 
in depressed patients and healthy controls. In general, patients and controls had similar patterns of decision making. Likewise, affective responses to the outcome of the decision and participants' ratings about the quality of their decision prior to the outcome were similar between the groups. This speaks against the notion that the groups differ in terms of a nonspecific "blunting" of task-related feedback or that differences in the points of reference subjects use to evaluate the outcomes of decisions can easily account for the pattern of results on this task. However, whereas control subjects showed a robust reduction in affective ratings if the nonobtained outcome was greater than the obtained outcome, as compared with when the obtained outcome was greater than or equal to the nonobtained outcome, depressed patients' affective reports showed little evidence of modulation by the value of the nonobtained outcome. This was particularly evident in subjects with greater self-reported apathy scores, particularly the emotional apathy subscale. The pattern of results is consistent with the notion that depressed patients show attenuated regret about the consequences of their decision-making performance.

These findings are consistent with our hypothesis that a representation of the response performed on a given trial, which might be disrupted in apathetic subjects, is critical for the normal experience of regret, as was emphasized by Zeelenberg et al. (2000). On the other hand, disappointment is better associated with contexts in which there is a sense of a lack of control (Zeelenberg, van Dijk, Manstead, \& van der Pligt, 1998), and in the present study, the obtained outcome, over which the subjects' control was often only partial, prompted emotional reactions of similar magnitude and valence between the groups. The critical effect of apathy appears to be reflected better in the manner in which feedback informs the subjects of their own decision-making strategy/performance, which we would expect to be most clearly manifest to the subjects in the comparison of the obtained and nonobtained outcomes.

It would have been difficult to anticipate the pattern of these results purely on the basis of existing literature about the neural representation of reinforcement in major depression. A variety of effects have been observed with different paradigms, which were briefly discussed in the introduction. Typically, the paradigms that are employed follow a straightforward Pavlovian format in which a stimulus precedes a rewarding outcome (e.g., Kumar et al., 2008) or are analyzed with a direct focus on important parameters, such as selection, anticipation, and outcome (e.g., Chiu \& Deldin, 2007; Knutson et al., 2008; Smoski et al., 2009). Collectively, these findings have so far resisted a straightforward unifying account and are generally not discussed in terms of regret or counterfactual cognition.

However, the neuropsychological literature might offer more insight. Gomez Beldarrain, Garcia-Monco, Astigarraga, Gonzalez, and Grafman (2005) observed a reduction of counterfactual thinking in prefrontal lesion patients with symptoms of depression. In addition, the correspondence between apathy and regret mirrors a reported rela- tionship between the integrity of the orbitofrontal cortex (OFC) and apathy: Apathy, although not clinical depression (Koenigs et al., 2008), is thought to be a symptom of medial prefrontal cortex damage (Fellows \& Farah, 2005; R. Levy \& Dubois, 2006), as well as other neurological disorders (M. L. Levy et al., 1998), while regret is also reduced following lesions of the OFC (Camille et al., 2004). Other lines of evidence suggest that the OFC, particularly its medial aspect, is critical for dimensions of the phenomenology of regret (Coricelli et al., 2005; Sommer, Peters, Glascher, \& Buchel, 2009). The OFC is thought to play a key role in the representation of decision parameters critical for normal decision making, and we therefore suggest that these might be disrupted in the dysfunctional OFC observed in depression (Drevets, 2007). The hypothesis that the OFC, rather than alternative candidates such as the anterior cingulate cortex or the hippocampus (Coricelli et al., 2005), might mediate an attenuation of regret in depressed/apathetic subjects awaits direct examination.

An intriguing aspect of apathy, identified by Fellows and Farah (2005), might shed light on the functional role of regret. They examined OFC lesion patients' and controls' ability to generate possible events in the future and were able to determine a time window that reflected the distance into the future that a given subject would be most likely to consider. Self-reported apathy reduced the temporal extent of this window. It may be that the experience of regret is dependent on the ability to think and plan into the future, being an important teaching signal (Roese, 1997), but one that is relevant only if the subject is thinking toward the future. Certainly, processing of information about the nonselected option suggests a desire to improve performance on future trials. Again, this hypothesis might be evaluated in future studies.

A negative bias in depression (Beck, 1967) may result from the generation of upward counterfactuals (states of the world that are more positive than current or predicted states). A predisposition to the formation of upward counterfactuals should render emotional ratings about the quality and outcomes of decisions generally more negative and should not, at least at first approximation, be selective for regret over disappointment, although a complex interaction effect on upward counterfactuals between controllability and depression severity has been observed (Markman \& Miller, 2006). Notably, a general negative movement of ratings was not observed in depressed patients, but our exploratory analysis showed that this was better associated with gender, where females were observed to show more negative ratings than did males. Implications for the importance of this finding for negative bias in depression are not clear, but at the very least, it shows that the paradigm is sensitive to a negative bias, which was not, however, associated with depression.

Limitations of this study should be acknowledged. First, the patients were medicated, but the controls were not. We argue that medication is unlikely to explain the entirety of our results, due to the selective influence of different symptoms. Nevertheless, it remains an important factor to consider in future studies. We chose to keep the number of trials small and gave a variety of different trial types to 
ensure that the subjects would remain engaged throughout the task, but future studies could usefully investigate the generality of the finding across a wider range of experimentally controlled parameters. In addition, a larger sample of patients would confirm that the null effects, especially the failure to observe effects of depression on the win/loss-related ratings and decision making, were veridi$\mathrm{cal}$. Indeed, in the case of the latter, prior literature suggests that such effects might be anticipated (Murphy et al., 2001; Smoski et al., 2008). Finally, it should be emphasized that although our operationalization of regret is useful, since it is easy to instantiate in an experimental paradigm, the extent to which it reflects the entire phenomenology of regret is not clear. In the context of the present study, our inference about the nature of disappointment and regret was based on the conditions required to elicit them (following, e.g., Mellers, Schwartz, \& Ritov, 1999), rather than on two separate interrogations of each emotion using different questions. We are currently investigating different measures of regret, including assessing possible differences in the statements used in the rating scales.

\section{Summary}

In summary, depressed patients reported a reduced experience of regret, as compared with controls, an effect that was particularly related to self-reported apathy. Gender affected baseline affective ratings to the outcome of the decision and ratings of decision quality, with females showing lower overall ratings than did males, but did not affect the magnitude of emotional response to the outcome or of regret. We propose that apathy reduces regret via the disruption of action representation in the OFC.

\section{AUTHOR NOTE}

This work was funded by a Wellcome Trust Programme Grant to T.W.R., B.J.S., B. J. Everitt, and A. C. Roberts, and was completed within the University of Cambridge Behavioural and Clinical Neuroscience Institute, supported by a consortium award from the Medical Research Council (U.K.) and the Wellcome Trust. H.W.C. was supported by a 3-year MRC studentship while this work was carried out. N.C. was supported by the Fondation Fyssen. Correspondence concerning this article should be addressed to H. W. Chase, School of Psychology, University of Nottingham, University Park, Room B60 Psychology, Nottingham NG7 2RD, England (e-mail: henry.chase@nottingham.ac.uk).

\section{REFERENCES}

Abramson, L. Y., Metalsky, G. I., \& Alloy, L. B. (1989). Hopelessness depression: A theory-based subtype of depression. Psychological Review, 96, 358-372.

American Psychiatric Association (1994). Diagnostic and statistical manual of mental disorders: DSM-IV (4th Ed.). Washington, DC: Author.

Beck, A. T. (1967). Depression: Clinical, experimental, and theoretical aspects. New York: Harper \& Row.

Beck, A. T., Epstein, N., Brown, G., \& Steer, R. A. (1988). An inventory for measuring clinical anxiety: Psychometric properties. Journal of Consulting \& Clinical Psychology, 56, 893-897.

Beck, A. T., Ward, C., \& Mendelson, M. (1961). Beck Depression Inventory (BDI). Archives of General Psychiatry, 4, 561-571.

Camille, N., Coricelli, G., Sallet, J., Pradat-Diehl, P., Duhamel, J. R., \& Sirigu, A. (2004). The involvement of the orbitofrontal cortex in the experience of regret. Science, 304, 1167-1170.

Chase, H. W., Frank, M. J., Michael, A., Bullmore, E. T., SahaKIAN, B. J., \& RobBINS, T. W. (2009). Approach and avoidance learn- ing in patients with major depression and healthy controls: Relation to anhedonia. Psychological Medicine, 40, 433-440.

Chiu, P. H., \& Deldin, P. J. (2007). Neural evidence for enhanced error detection in major depressive disorder. American Journal of Psychiatry, 164, 608-616.

Coricelli, G., Critchley, H. D., Joffily, M., O’Doherty, J. P., Sirigu, A., \& Dolan, R. J. (2005). Regret and its avoidance: A neuroimaging study of choice behavior. Nature Neuroscience, 8, 1255 1262 .

Coricelli, G., Dolan, R. J., \& Sirigu, A. (2007). Brain, emotion and decision making: The paradigmatic example of regret. Trends in $\mathrm{Cog}$ nitive Sciences, 11, 258-265.

Drevets, W. C. (2007). Orbitofrontal cortex function and structure in depression. In G. Schoenbaum, J. A. Gottfried, \& E. A. Murray (Eds.), Linking affect to action: Critical contributions of the orbitofrontal cortex (Annals of the New York Academy of Sciences, Vol. 1121, pp. 499-527). New York: New York Academy of Sciences.

Fellows, L. K., \& FARAH, M. J. (2005). Dissociable elements of human foresight: A role for the ventromedial frontal lobes in framing the future, but not in discounting future rewards. Neuropsychologia, 43, $1214-1221$

Gomez Beldarrain, M., Garcia-Monco, J. C., Astigarraga, E., Gonzalez, A., \& Grafman, J. (2005). Only spontaneous counterfactual thinking is impaired in patients with prefrontal cortex lesions. Cognitive Brain Research, 24, 723-726.

Holmes, A. J., \& Pizzagalli, D. A. (2008). Spatiotemporal dynamics of error processing dysfunctions in major depressive disorder. Archives of General Psychiatry, 65, 179-188.

Kahneman, D., \& Tversky, A. (1979). Prospect theory: An analysis of decision under risk. Econometrica. 47, 263-291.

Knutson, B., Bhanji, J. P., Cooney, R. E., Atlas, L. Y., \& Gotlib, I. H. (2008). Neural responses to monetary incentives in major depression. Biological Psychiatry, 63, 686-692.

Koenigs, M., Huey, E. D., Calamia, M., Raymont, V., Tranel, D., \& Grafman, J. (2008). Distinct regions of prefrontal cortex mediate resistance and vulnerability to depression. Journal of Neuroscience, 28, 12341-12348.

Kumar, P., Waiter, G., Ahearn, T., Milders, M., Reid, I., \& Steele, J. D. (2008). Abnormal temporal difference reward-learning signals in major depression. Brain, 131, 2084-2093.

Larsen, J. T., McGraw, A. P., Mellers, B. A., \& Cacioppo, J. T. (2004). The agony of victory and thrill of defeat: Mixed emotional reactions to disappointing wins and relieving losses. Psychological Science, 15, 325-330.

Levy, M. L., Cummings, J. L., Fairbanks, L. A., Masterman, D., Miller, B. L., Craig, A. H., ET AL. (1998). Apathy is not depression. Journal of Neuropsychiatry \& Clinical Neuroscience, 10, 314-319.

Levy, R., \& Dubois, B. (2006). Apathy and the functional anatomy of the prefrontal cortex-basal ganglia circuits. Cerebral Cortex, 16, 916928.

Marin, R. S. (1996). Apathy: Concept, syndrome, neural mechanisms, and treatment. Seminars in Clinical Neuropsychiatry, 1, 304-314.

Marin, R. S., BiedrzyCKI, R. C., \& Firinciogullari, S. (1991). Reliability and validity of the Apathy Evaluation Scale. Psychiatry Research, 38, 143-162.

Markman, K. D., \& Miller, A. K. (2006). Depression, control, and counterfactual thinking: Functional for whom? Journal of Social \& Clinical Psychology, 25, 210-227.

Mellers, B. A. (2000). Choice and the relative pleasure of consequences. Psychological Bulletin, 126, 910-924.

Mellers, B. [A.], Schwartz, A., \& Ritov, I. (1999). Emotion-based choice. Journal of Experimental Psychology: General, 128, 332-345.

Monroe, M. R., Skowronski, J. J., MacDonald, W., \& Wood, S. E. (2005). The mildly depressed experience more post-decisional regret than the non-depressed. Journal of Social \& Clinical Psychology, 24, 665-690.

Montgomery, S. A., \& Asberg, M. (1979). A new depression scale designed to be sensitive to change. British Journal of Psychiatry, 134, 382-389.

Murphy, F. C., Rubinsztein, J. S., Michael, A., Rogers, R. D., Robbins, T. W., Paykel, E. S., \& Sahakian, B. J. (2001). Decisionmaking cognition in mania and depression. Psychological Medicine, 31, 679-693. 
Nelson, H., \& Willison, J. (1991). National Adult Reading Test (NART) test manual. Windsor, UK: NFER-Nelson.

Pizzagalli, D. A., Holmes, A. J., Dillon, D. G., Goetz, E. L., Birk, J. L., Bogdan, R., ET AL. (2009). Reduced caudate and nucleus accumbens response to rewards in unmedicated individuals with major depressive disorder. American Journal of Psychiatry, 166, 702-710.

Pizzagalli, D. A., Jahn, A. L., \& O'SheA, J. P. (2005). Toward an objective characterization of an anhedonic phenotype: A signal-detection approach. Biological Psychiatry, 57, 319-327.

Roese, N. J. (1997). Counterfactual thinking. Psychological Bulletin, 121, 133-148.

Rogers, R. D., Everitt, B. J., Baldacchino, A., Blackshaw, A. J., Swainson, R., Wynne, K., ET AL. (1999). Dissociable deficits in the decision-making cognition of chronic amphetamine abusers, opiate abusers, patients with focal damage to prefrontal cortex, and tryptophan-depleted normal volunteers: Evidence for monoaminergic mechanisms. Neuropsychopharmacology, 20, 322-339.

Smoski, M. J., Felder, J., Bizzell, J., Green, S. R., Ernst, M., LynCh, T. R., \& Dichter, G. S. (2009). fMRI of alterations in reward selection, anticipation, and feedback in major depressive disorder. Journal of Affective Disorders, 118, 69-78.

Smoski, M. J., Lynch, T. R., Rosenthal, M. Z., Cheavens, J. S., Chapman, A. L., \& Krishnan, R. R. (2008). Decision-making and risk aversion among depressive adults. Journal of Behavior Therapy \& Experimental Psychiatry, 39, 567-576.

Sommer, T., Peters, J., Glascher, J., \& Buchel, C. (2009). Structurefunction relationships in the processing of regret in the orbitofrontal cortex. Brain Structure \& Function, 213, 535-551.

Steele, J. D., Kumar, P., \& Ebmeier, K. P. (2007). Blunted response to feedback information in depressive illness. Brain, 130, 2367-2374.

Zeelenberg, M., van Dijk, W. W., Manstead, A. S. R., \& van der Pligt, J. (1998). The experience of regret and disappointment. Cognition \& Emotion, 12, 221-230.

Zeelenberg, M., van DiJK, W. W., Manstead, A. S. R., \& VAn DeR Pligt, J. (2000). On bad decisions and disconfirmed expectancies: The psychology of regret and disappointment. Cognition \& Emotion, 14, 521-541.

\section{SUPPLEMENTAL MATERIALS}

A supplemental figure depicting the task from this article may be downloaded from http://cabn.psychonomic-journals.org/content/ supplemental.

(Manuscript received July 28, 2009; revision accepted for publication February 11, 2010.) 